\title{
New Synthesis-Regulatory Evolution, the Veiled World of Chemical Diversification
}

\author{
Daniel J. Kliebenstein
}

Published online: 20 March 2013

(C) Springer Science+Business Media New York 2013

Chemical Ecology focuses on how chemicals mediate interactions among organisms. This role of chemistry in organismal interactions generates an evolutionary pressure of pests and hosts to enter into a repetitive cycle of defense novelization and counter-response. This cycle provides the central evolutionary hypothesis about the pressure to derive novel chemicals and thus generate a massive chemical diversification among and within plant and fungal lineages. In the past decades, significant efforts from a large research community have made great progress in cloning numerous enzymes from diverse biochemical pathways leading to a general genomic thesis for this chemical diversification. This thesis can be stated as follows: genes duplicate either individually or as whole genomes. These duplicated genes can either "neo-functionalize" (evolve completely new biochemical functions) or "sub-functionalize" (evolve a fraction of the original function). It is the presence of these changed duplicated genes that provides new enzymatic capacity that can somehow be coordinated into a single pathway to produce a single compound.

However, this gene evolution model and the data supporting it focus almost entirely on how the enzymatic capacity to make a new compound arises, while saying little about the regulatory processes that may have accompanied this evolution. This is despite the fact that such regulatory processes are essential to coordinate the pathway and allow it to function. It is possible that part of this lack of enquiry into regulatory evolution comes from the common observation that most secondary metabolite pathways show similar inductive regimes in response to pathogens or herbivores, generating the illusion that they may involve conserved regulators that trap the biochemical pathway promoters. However, recent work is suggesting that there is as much regulatory evolution at play in chemical diversification as there is enzyme evolution.

A common observation in secondary metabolism is that biosynthetic pathways frequently are controlled by bHLH and MYB transcription factors. A recent multi-species genomic phylogenetic analysis of the bHLH and MYB families showed that like secondary metabolite enzymes, these families have undergone a massive expansion within the vascular plants (Feller et al., 2011). There were similar levels of gene numbers as for key secondary metabolite enzymes like CYP450s and 2ODDs, and there was even a similar propensity for lineagespecific families of both bHLH and MYB genes.

One of these lineage-specific MYB families includes those MYBs that control the expression of glucosinolates, amino acid derived defense compounds present in the Capparales. A recent investigation of how the glucosinolate enzymes and their affiliated MYBs evolved in the Capparales showed that early in their evolutionary history, a full biosynthetic pathway along with a specific MYB evolved to create a functioning glucosinolate system (Bekaert et al., 2012). During Capparales evolution, there were whole genome duplication events that duplicated

D. J. Kliebenstein $(\bowtie)$

Department of Plant Sciences, University of California, Davis,

Davis, CA 95616, USA

e-mail: kliebenstein@ucdavis.edu

URL: www.plantsciences.ucdavis.edu/kliebnestein the entire glucosinolate pathway and the MYBs. Following one of these duplications, the genes partitioned into largely two pathways, one for making glucosinolates from Tryptophan and the other using Methionine. Central to the issue of regulatory evolution, the MYBs also split along downstream pathway lines. Thus, as suggested by the phylogenetic analysis, chemical diversification also is likely driving regulatory factor diversification within plants.

Intriguingly, recent work suggests that secondary metabolite transcription factors are not limited to a role in secondary metabolism. Analysis of volatile production and regulation within Petunia identified the missing link in a regulatory cascade of MYBs. In this system, three MYBs (EOBII, EOBI, and $O D O 1$ ) form a hierarchical regulatory cascade leading to the production of volatiles in Petunia (Spitzer-Rimon et al., 2013). These MYBs also directly regulated primary metabolite genes within the shikimate pathway, presumably controling precursor availability for the production of benzenoids. Importantly, these genes are not broadly evolutionarily conserved and appear to have arisen specifically in this lineage for secondary volatile production. This function in regulating secondary metabolism, however, did not prevent them from accruing regulatory control over a primary metabolite pathway like the shikimate pathway. Thus, secondary metabolite regulatory factors may also evolve roles in mediating primary metabolic or physiological processes as a part of coordinating secondary metabolite production, in this case regulating precursor availability.

Together, these works suggest that the chemical diversification so central to the expansion of biochemical capacity is also driving an unrecognized expansion of regulatory capacity within vascular plants. More dramatically, if lineagespecific secondary metabolite transcription factors frequently acquire the capacity to control central physiological processes that enable the optimization of chemical defenses, then the chemical diversification central to Chemical Ecology may play a fundamental role in shaping areas outside the field's traditional boundaries, such as core plant physiology and metabolism. In either case, studying how chemical diversification and regulatory evolution have proceeded in coordination will provide unique insight into how organisms and their networks evolve.

\section{References}

Bekaert, M., Edger, P. P., Hudson, C. M., Pires, J. C., and Conant, G. C. 2012. Metabolic and evolutionary costs of herbivory defense: Systems biology of glucosinolate synthesis. New Phytol. 196(2):596-605.

Feller, A., Machemer, K., Braun, E. L., and Grotewold, E. 2011. Evolutionary and comparative analysis of MYB and bHLH plant transcription factors. Plant J. 66(1):94-116.

Spitzer-Rimon, B., Farhi, M., Albo, B., Cna'ani, A., Ben Zvi, M. M., Masci, T., Edelbaum, O., Yu, Y., Shklarman, E., Ovadis, M., Et AL. 2013. The R2R3-MYB-like regulatory factor EOBI, acting downstream of EOBII, regulates scent production by activating ODO1 and structural scent-related genes in petunia. Plant Cell. doi:10.1105/tpc.112.105247. 